\title{
PID regulator on thermal power simulation machine
}

\author{
Ming Zhang ${ }^{1, a}$, Yuming Huang ${ }^{2, b}$ \\ ${ }^{1,2}$ Zhejiang Energy Group R\&D, Zhejiang, China \\ amingzhang@gmail.com, byuminghuang@gmail.com
}

Keywords: PID; simulation machine; control algorithm; thermal power units

\begin{abstract}
For the reality of thermal power units and the training needs of operating crew, introduce the PID regulator on the thermal power plant simulation machine. Depending on the various needs of simulation modeling, introduce the PID regulator control algorithm and some auxiliary functions. Through simulation of the actual unit, Verify that the controller has good dynamic performance and robustness.
\end{abstract}

\section{Introduction}

In the historical development of the automatic control of production process, PID control is the oldest and most energetic basic control method. Because PID control has a simple principle and is convenient, adaptable and robust, it has been widely used in electrical production. Large-scale thermal power plant occupies a dominant position in China's electric power industry. A large-scale thermal power generating units has the characteristics of high efficiency, high level of automation, and so on, so the quality requirements to operating crew are relatively high. The traditional apprenticeship has been difficult to meet the actual needs of the field production. Therefore, the simulator for thermal power generating unit plays an increasingly important role in electrical production.

At present, the simulator for thermal power generating unit is increasingly used for training line personnel in power plant operation and make them master of startup, shutdown, and monitor, operation, and processing in normal or accident conditions. These trainings become realized through monitoring and control system on the simulator. Therefore, the control system simulation is important as the same as the simulation for primary device and main system of power unit, and is an important part of the simulation system of power plant. Although DCS is used extensively in the automatic control system of power plant, but as for its control strategy, the classic PID algorithm is dominant. therefore, the PID regulator simulation also plays an important role in the control system simulation.

\section{Control algorithm of PID regulator}

A regulator which is controlled in accordance with proportional, integral and derivative is called PID regulator for short. PID regulator is the most widely used kind of control and regulation laws in industrial processing control systems[2]. Not only can it break down to proportional control(P), proportional integral control (PI) and proportional derivative control (PD) based on the actual needs of the scene, but also some PID regulators can be combined into several complex controls, such as cascade control, ratio control and so on[3].

An control law for ideal continuous PID control law can be described in formula like this:

$$
\mathrm{u}(\mathrm{t})=\mathrm{K}_{\mathrm{p}}\left[\mathrm{e}(\mathrm{t})+\frac{1}{\mathrm{~T}_{\mathrm{i}}} \int_{0}^{t} e(\mathrm{t}) \mathrm{dt}+\mathrm{T}_{\mathrm{d}} \frac{d e(t)}{d t}\right]+u_{0}
$$

In the formula, $\mathrm{e}(\mathrm{t})$ is the input deviation; $u(t)$ is the output of control process; $K_{p}$ is the proportional gain; $T_{i}$ is the integral time; $T_{d}$ is the derivative time; $u_{0}$ is the initial control value.

In order to make the computer calculate the formula, we must be convert a continuous PID control algorithm into a discrete PID control algorithm. For every sampling period which is set to $T_{s}$, 
conduct once data sampling, control system operation and data output. The discrete PID control algorithm can be derived from a continuous ideal PID control algorithm through discretization directly. Use the following relational expression for discretization[4]:

$$
\left\{\begin{array}{l}
\int_{0}^{t} e(t) d t \approx T_{s} \sum_{i=0}^{k} e(i) \\
\frac{d e(t)}{d t} \approx \frac{e(k)-e(k-1)}{T_{s}}
\end{array}\right.
$$

So discrete PID control algorithm can be expressed as the following equation:

$$
u(k)=K_{p}\left\{\mathrm{e}(\mathrm{k})+\frac{T_{s}}{T_{i}} \sum_{i=0}^{k} e(i)+\frac{T_{d}}{T_{s}}[e(k)-e(k-1)]\right\}+u_{0}
$$

$\sum_{i=0}^{k} e(i)$ related to all the states in the past, tedious calculation and taking up too much memory, which are why use the incremental algorithm, $\Delta u(k)=u(k)-u(k-1)$. Obtain a formula after simplification:

$$
\Delta u(k)=K_{p} \Delta e(k)+K_{I} e(k)+K_{D}[e(k)-2 e(k-1)+e(k-2)]
$$

In the formula, $\Delta \mathrm{e}(\mathrm{k})=\mathrm{e}(\mathrm{k})-\mathrm{e}(\mathrm{k}-1) ; K_{I}=\frac{K_{P} T_{S}}{T_{i}} ; K_{D}=\frac{K_{P} T_{d}}{T_{s}}$.

In addition, for some of the control features of the simulation machine, do some processing as follows.

1) Due to the PID controller output direct drive has a actuator module with proportional characteristic, and it needs to track the actual valve location during the period of manual control, so using the integral incremental type position control algorithm smooths the deviations integral terms.

2) The derivative action of ideal PID equation for the response of differential effect of high frequency interference is too sensitive. It's prone to oscillate in control process and so adopting incomplete derivation. Derivative action in a long time keeps the equal condition on the trend of deviation change. It can improve the quality of control system.

3) proportional, integral and derivative action are independent of each other, to allow operators to directly understand and adjust the control parameters on the control effect.

Therefore, the control algorithm that PID controller module actually using is:

$$
u(k)=u_{p}(k)+u_{i}(k)+u_{d}(k)
$$

In that, proportional term is $u_{p}(k)=K_{p} e(k)$;

The integral term is $u_{i}(k)=u_{i}(k-1)+K_{p} \frac{T_{s}}{2 T_{i}}[e(k)+e(k-1)]$;

The derivative term is $\frac{T_{d}}{T_{s}+T_{d}}\left\{u_{d}(k-1)+K_{d}[e(k)-e(k-1)]\right\}$;

$K_{d}$ is the Differential amplification coefficient.

\section{Establish a simulation model for PID regulator}

Because PID controller module is a composite module, the structure also took into account a number of other auxiliary functions.

1) Set an output limits, ensuring that the output signal is in the allowable range.

2) Set a polarity switch, for user-friendly establishment of PID controller to the polarity relationship between input deviation and output control.

3) Set an automatic and manual switch, to achieve undisturbed switching of control mode and automatic tracking of control algorithms. When set to automatic, the output is the result by PID control algorithm for computing input deviation : $u(k)=u_{p}(k)+u_{i}(k)+u_{d}(k)$. When set to manual, 
the output is the racking data: $u(k)=x_{i}(k) ; u_{i}(k)=x_{i}(k)-u_{p}(k)-u_{d}(k)$. In the formula, $x_{i}(k)$ is the tracking signal.

4) Set a feedforward part. For large delay and multi-interference controlled plant, increase feedforward control function in the control loop. The feedforward controlled variable is $u_{f}(k)=K_{f} x_{f}(k)$, where, $K_{f}$ is a feedforward coefficient and $x_{\mathrm{f}}(\mathrm{k})$ is a feedforward signal. The formula of Feedforward-feedback compound control is:

$$
\mathrm{u}(\mathrm{k})=\mathrm{u}_{\mathrm{p}}(\mathrm{K})+\mathrm{u}_{\mathrm{i}}(\mathrm{k})+\mathrm{u}_{\mathrm{d}}(\mathrm{k})+\mathrm{u}_{\mathrm{f}}(\mathrm{k})
$$

5) Set a dead zone. That is a process control system which allows certain variable to change within limits which is help to avoid frequent action of executive component.

\section{Simulation example}

Apply the above PID functions to the actual 350 MW thermal power plant simulation machine. The simulator supports the whole process from model development, debugging, operation to analysis. According to control schematic diagram or configuration diagram of the actual unit from the plant, select the appropriate simulation models to connect configuration, and the appropriate parameter value for initialization to achieve a specific control system simulation

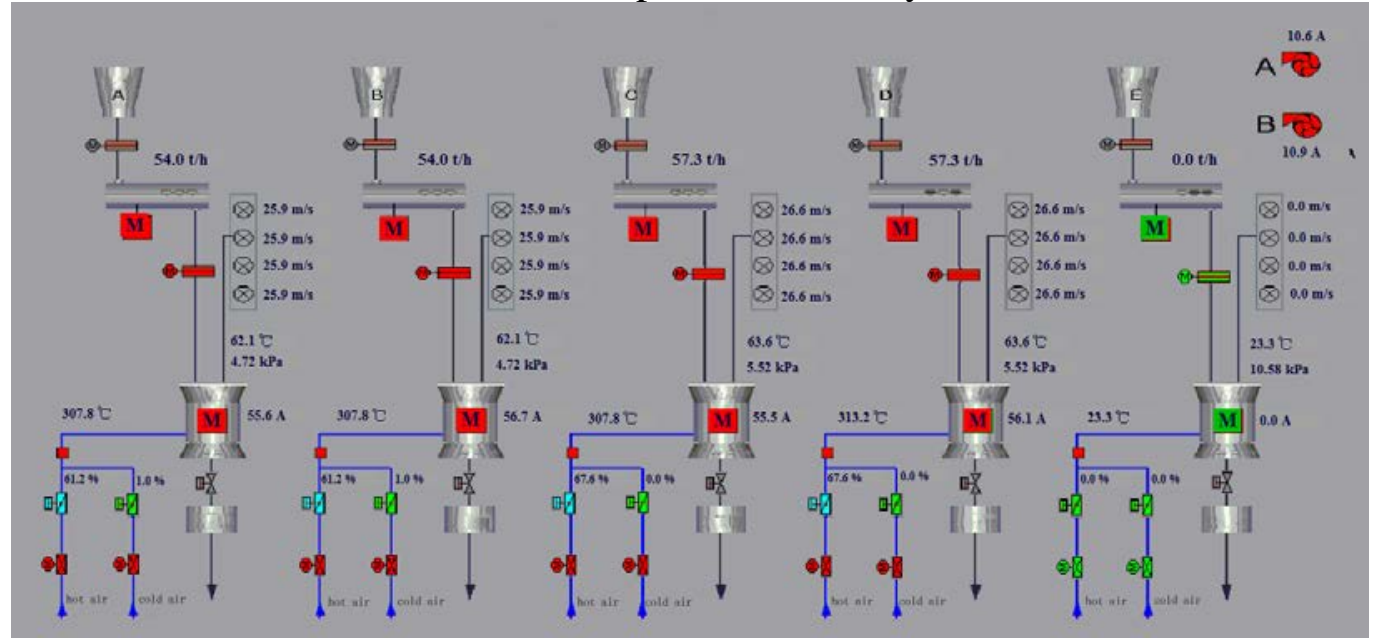

Fig $1350 \mathrm{MW}$ thermal power plant simulation

After building the simulation loop, impose $10 \%$ of the load disturbance on the DEH control system of steam turbine, to observe the control characteristics of power control loop, as shown in fig 2. After imposing a step disturbance on the high water level, we can observe a characteristic that the level returns from $320 \mathrm{~mm}$ to the presupposed $200 \mathrm{~mm}$, as shown in fig 3.

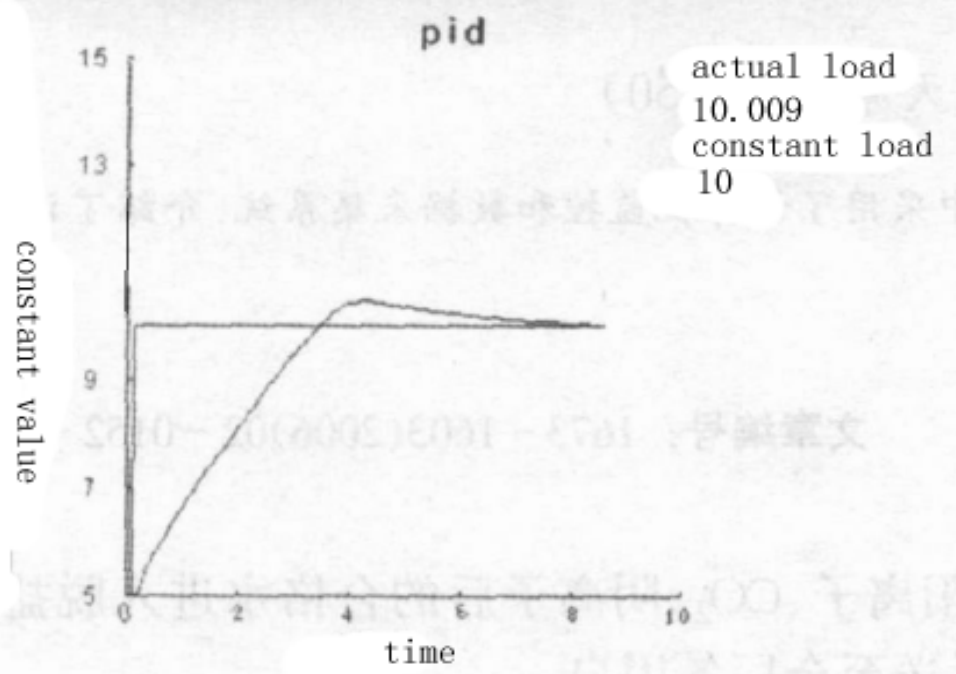

Fig 2 the control characteristics of load disturbance in $10 \%$ 


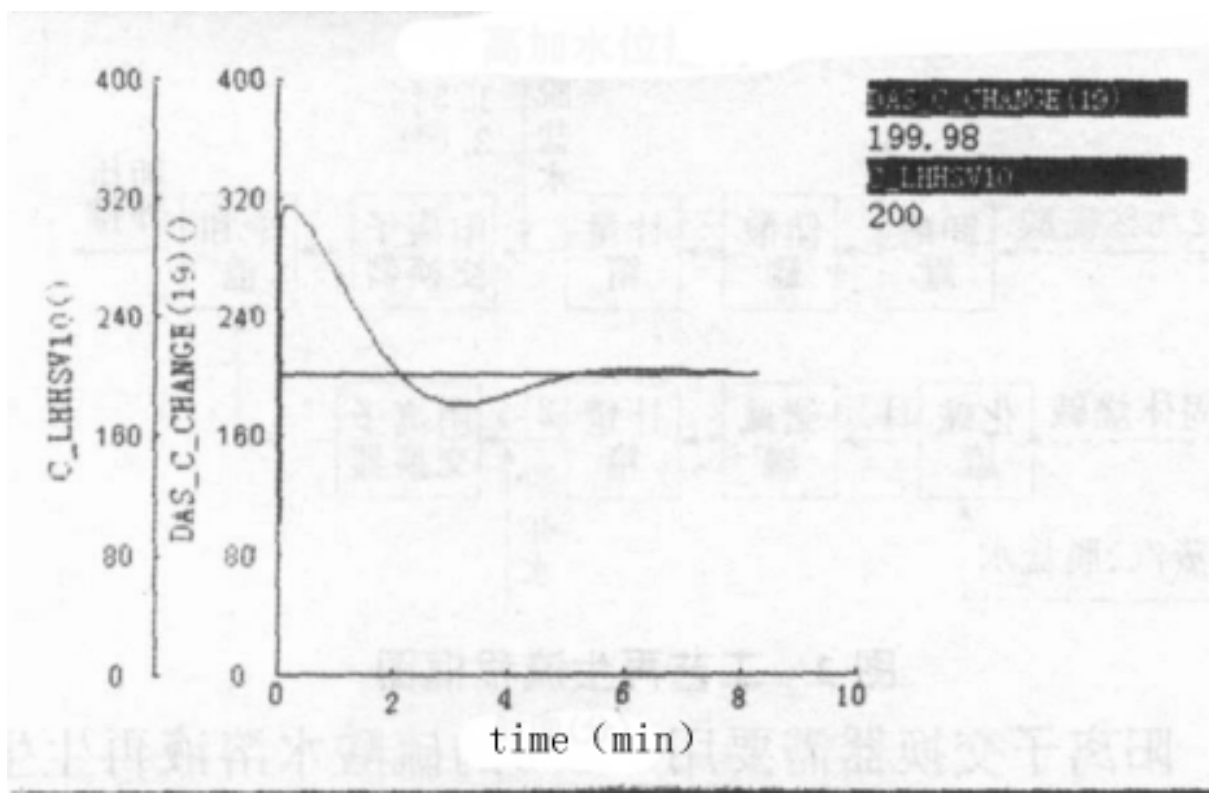

Fig 3 high water level control

\section{Conclusion}

The above simulation shows that PID regulator algorithm can meet the technical requirements of thermal power simulator. This modeling approach was applied in the simulation process of $350 \mathrm{MW}$ thermal power units. The simulation machine get all the praise from the plant operating personnel, and it can reflect the dynamic characteristics of the actual control system.

\section{References}

[1] M.K.Rangla, Simulation of activated sludge process control strategies. Simulation '98. International Conference on (Conf. Publ. No. 457), 30 Sep-2 Oct 1998, Pages(s):p152 157.

[2] Wang Ming-chun, Design of Distributed Simulator System for Fossil Fired Power Generating Unit, Automation of Electric Power Systems. 2002.26(3):p66 68.

[3] Leng Wei, Fang De-shan, Xu Zhi-gao, Application and Development of Fossil Power Plant Simulation. Automation of Electric Power Systems, 1999, 23 (23):p7 14.

[4] Duan Xin-hui. Development of DCS Configuration Text Automatic Correspond to Control System Simulation Model Software Used in Power Station. 2003,20(1): p102 105.

[5] O. S. Kwon and H. Heo, MIMO controller with compensator via gain tuning method for steam temperature control of thermal power plant, Proc. of the International Multi Conference on Complexity, Information and Cybernetics, Orlando, Florida, USA. (2010) p329-334.

[6] O.-Shin Kwon, Won-Hee Jung, Hoon Heo, Steam temperature controller with LS-SVR-based predictor and PID gain scheduler in thermal power plant, Journal of Mechanical Science and Technology, 2013, Vol.27, p557-565

[7] Rabindra Kumar Sahu, Sidhartha Panda, Pratap Chandra Pradhan, Design and analysis of hybrid firefly algorithm-pattern search based fuzzy PID controller for LFC of multi area power systems, International Journal of Electrical Power and Energy Systems, 2015, Vol.69, p200-212.

[8] Sanjoy Debbarma, Lalit Chandra Saikia, Nidul Sinha, Automatic generation control using two degree of freedom fractional order PID controller, International Journal of Electrical Power \& Energy Systems, 2014, Vol.58, p120-129 
[9] Rabindra Kumar Sahu, Sidhartha Panda, Narendra Kumar Yegireddy, A novel hybrid DEPS optimized fuzzy PI/PID controller for load frequency control of multi-area interconnected power systems, Journal of Process Control, 2014, Vol.24, p1596-1608

[10]Jiangjiang Wang, Dawei An, Chunfa Zhang, Youyin Jing, Genetic optimization algorithm of PID decoupling control for VAV air-conditioning system, Transactions of Tianjin University, 2009, Vol.15, p308-314 